\title{
TFF3 Is a Normal Breast Epithelial Protein and Is Associated with Differentiated Phenotype in Early Breast Cancer but Predisposes to Invasion and Metastasis in Advanced Disease
}

\author{
Ahmed R.H. Ahmed, ${ }^{* \dagger}$ Andrew B. Griffiths, ${ }^{\neq}$ \\ Michael T. Tilby, ${ }^{\star}$ Bruce R. Westley, ${ }^{\star \S}$ and \\ Felicity E.B. May ${ }^{\star}$ \\ From the Northern Institute for Cancer Research,* Faculty of \\ Medical Sciences, and the Department of Pathology, University \\ of Newcastle upon Tyne, Newcastle upon Tyne, United Kingdom, \\ the Department of Pathology, ${ }^{\dagger}$ Faculty of Medicine, Sohag \\ University, Sohag, Egypt; and the Breast Cancer Unit, \\ Department of Surgery, Royal Victoria Infirmary, Newcastle upon \\ Tyne, United Kingdom
}

The trefoil protein TFF3 stimulates invasion and angiogenesis in vitro. To determine whether it has a role in breast tumor metastasis and angiogenesis, its levels were measured by immunohistochemistry in breast tissue with a specific monoclonal antibody raised against human TFF3. TFF3 is expressed in normal breast lobules and ducts, at higher levels in areas of fibrocystic change and papillomas, in all benign breast disease lesions, and in $89 \%$ of in situ and in $83 \%$ of invasive carcinomas. In well-differentiated tumor cells, TFF3 is concentrated at the luminal edge, whereas in poorly differentiated cells polarity is inverted and expression is directed toward the stroma. Expression was high in well-differentiated tumors and was associated significantly with low histological grade and with estrogen and progesterone receptor expression, accordant with induction of TFF 3 mRNA by estrogen in breast cancer cells. Paradoxically, TFF3 expression was associated with muscle, neural, and lymphovascular invasion and the presence and number of involved lymph nodes, and it was an independent predictive marker of lymphovascular invasion and lymph node involvement. Consistent with an angiogenic function, TFF 3 expression correlated strongly with microvessel density evaluated with CD31 and CD34. In conclusion, TFF3 is expressed in both the normal and diseased breast. Although associated with features of good progno- sis, its profile of expression in invasive cancer is consistent with a role in breast tumor progression and tumor cell dissemination. (Am J Pathol 2012, 180: 904-916; DOI: 10.1016/j.ajpath.2011.11.022)

Improvements in the early diagnosis and clinical management of breast cancer have led to an impressive reduction in mortality notwithstanding which $>500,000$ people die every year as a direct result of disseminated disease. During carcinogenesis, neoplastic cells acquire the ability to migrate, invade, and metastasize either directly into neighboring tissues or via the neural network, lymphatic system, or vasculature to more distant sites. In breast cancer, malignant cells can invade locally to the underlying muscle or overlying skin or can escape through the neural sheath or via the lymph or blood vessels. The presence of tumor metastases in the axillary lymph nodes is an important part of clinical staging and prediction of prognosis. It is accepted that the existence of metastases in regional lymph nodes is not a precursor of but a manifestation of more distant spread. Distant metastases occur most commonly in bone, followed by lung, liver, and brain. Understanding of the underlying biology of breast cancer and breast cancer metastasis remains obscure yet is increasingly relevant clinically with the move toward stratified medicine and therapeutic monitoring.

Systemic therapies are important in the treatment of both early stage and advanced breast cancers. Endocrine therapies, which have been the mainstay of breast cancer therapy, exploit the dependence of tumor cells on estrogens and include partial and pure antiestrogens and

Supported by the Egyptian Government Mission through an Overseas Fellowship (A.R.H.A.).

Accepted for publication November 17, 2011

Address reprint requests to Felicity E.B. May, D.Phil., Northern Institute for Cancer Research, Department of Pathology, Faculty of Medical Sciences, University of Newcastle upon Tyne, Framlington Place, Newcastle upon Tyne, NE2 4HH, UK. E-mail: F.E.B.May@ncl.ac.uk. 
type I and type II aromatase inhibitors. Tumors that are likely to respond to endocrine therapies are identified by the presence of the estrogen receptor $\alpha$, which is a requisite for estrogen action, and the progesterone receptor, which is induced by estrogen.

Trefoil proteins are small, secreted proteins that are co-expressed with mucins by epithelia and are thought to be important in normal mucosal protection and repair. ${ }^{1,2}$ The three human members of the trefoil factor family, TFF1, TFF2, and TFF3, are characterized by the presence of a conserved 41- to 42-amino acid residue trefoil domain with distinctive structure. TFF1 and TFF3 contain one trefoil domain, whereas TFF2 contains two. TFF3 has an extra trefoil domain cysteine residue that forms intermolecular disulfide bonds. ${ }^{3}$ The structure of dimeric TFF3 is considerably more compact than that of dimeric TFF1. ${ }^{3-5}$

The normal functions of trefoil proteins are thought to center on their role in mucosal protection, namely interactions with mucins and stimulation of cell motility. ${ }^{1,2}$ TFF3 has been found to stimulate cellular invasion in vitro and to have angiogenic activity. 6,7 The role of trefoil proteins in cancer is controversial. TFF1 is thought to be a tumor suppressor in gastric cancer, ${ }^{8}$ whereas in other tumors, including breast, prostate, and bladder, trefoil factor expression has been considered to be ectopic. ${ }^{1}$ Their expression has been reported to be predictive both of better survival $^{9,10}$ and of a more aggressive phenotype. ${ }^{11-14}$

TFF1 was discovered first as an abundant estrogenregulated molecule in breast cancer cells, whereas TFF2 is expressed only at very low levels in breast cancer cells. ${ }^{15-18}$ In 1997, TFF3 mRNA was detected by in situ hybridization in normal breast, in benign and in situ lesions, and in 31 invasive carcinomas $^{19}$ and was shown to be induced by estrogen and inhibited by antiestrogens in estrogen-responsive breast cancer cells. ${ }^{20}$ Subsequently, large expression microarray studies identified TFF3 as one of the genes whose expression is most closely associated with expression of estrogen receptor $\alpha .{ }^{21,22}$ The expression and role of TFF3 protein in human breast tissue have not been studied.

We report that TFF3 protein is expressed in the normal breast, benign breast disease, and in situ carcinoma and that its expression is lost in some invasive breast carcinomas. We provide evidence that, although TFF3 protein expression is higher in well-differentiated tumors and is associated with markers of estrogen responsiveness, TFF3 has a more sinister role in breast cancer invasion and metastasis.

\section{Materials and Methods}

\section{Collection of Tissue Samples}

Ethical permission for the studies was obtained from the Joint Newcastle Health Hospitals and University of Newcastle on Tyne Ethical Committee. Samples were collected from archived material of patients who presented sequentially between 2002 and 2004. Tissue was selected, and 1 -mm cores of tissue were embedded in tissue microarrays. Duplicate cores of tissue were taken from different blocks and arrayed in separate paraffin blocks. In total, 34 samples of normal breast, 86 benign breast lesions, 116 in situ carcinomas, 266 invasive carcinomas, and 76 involved lymph nodes were included in the study. In addition, whole sections of normal breast tissue from 40 women between the ages of 18 and 39 were analyzed.

\section{Immunohistochemistry}

Sections of formalin-fixed, paraffin-embedded breast tissue were deparaffinized and rehydrated. They were incubated in $0.5 \%$ hydrogen peroxide for 10 minutes to block endogenous peroxide activity. Antigen retrieval was with trypsin for 10 minutes at room temperature, in a $10 \mathrm{mmol} / \mathrm{L}$ citrate buffer, $\mathrm{pH} 6.0$, in a pressure cooker for 0.5 to 3 minutes or in a microwave for $2 \times 5$ minutes. After antigen retrieval, the tissue was washed in Tris- $\mathrm{HCl}, \mathrm{pH}$ 7.6, buffered saline and $1 \%$ Tween 20 . The tissues were incubated with a specific anti-TFF3 monoclonal antibody (manuscript in preparation) raised against correctly folded human TFF3 protein,, 3,4 a specific anti-TFF1 monoclonal antibody raised against correctly folded human TFF1 protein, ${ }^{23}$ anti-CD31 (JC70A; Dako UK Ltd., Ely, Cambridgeshire, UK), anti-D34 (QBend-10; Dako UK Ltd.), or anti-Ki-67 (MIB-1; Dako UK Ltd.) diluted in Trisbuffered saline and 1\% Tween 20 at room temperature and then with the Universal Probe [X-Cell Plus Polymer HRP (horseradish peroxidase) detection kit; A. Menarini Diagnostics, Firenze, Italy] at room temperature and finally with HRP-Polymer (A. Menarini Diagnostics). The brown colorimetric reaction was developed by incubation with 3,3' diaminobenzidine, and the sections were counterstained with hematoxylin.

\section{Scoring and Statistical Analysis}

The immunohistochemistry results were analyzed to generate a histoscore that combines the intensity of the immunoreaction with the number of positive cells. One thousand cells were scored individually as negative, weakly positive, moderately positive, or strongly positive. These four categories are weighted as $0,0.1,0.3$, and 1.0, respectively, in recognition of the range of intensities of the immunoreaction. The histoscores varied between 0 and 1000. A sample of normal human colonic mucosa was included in all immunohistochemical experiments to serve as a reference of the intensity of the immunoreaction. The final histoscore is the mean of scores from the two sections for each sample. The concordance between the two values was strong and significant (Spearman's $\rho$ correlation, $P<0.001$ ).

The results were analyzed statistically with the SPSS-PC package version 17 (SPSS Inc., Chicago, IL). Probabilities of $<0.05$ were considered statistically significant. The statistical significance of differences observed was analyzed by Kruskal-Wallis II or Mann-Whitney $U$ tests and of correlations by Pearson's correlation or Spearman's $\rho$ correlation. To adjust for potential confounding variables and to single out independent predictors, a multivariate analysis was undertaken. The different histoscores were scaled, and the predictive ability of the continuous vari- 

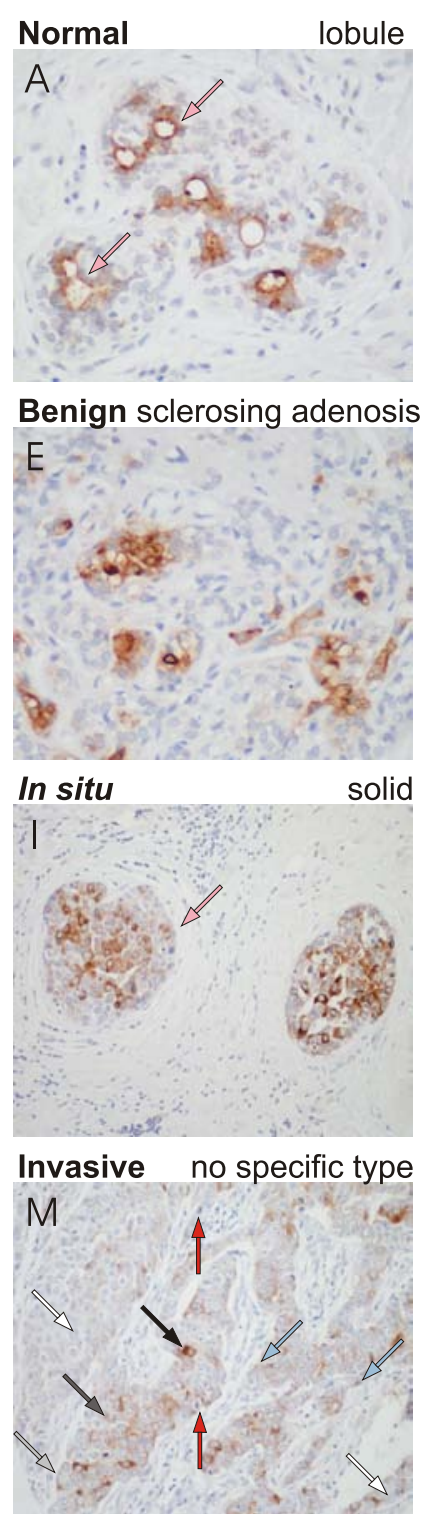

Invasive medullary

Q
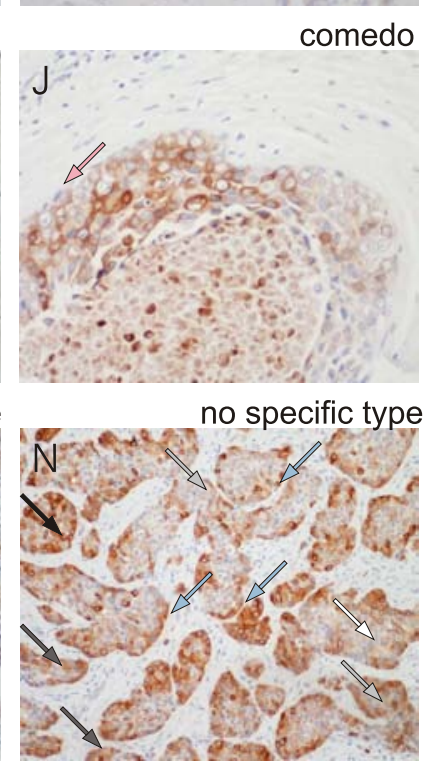

metaplastic
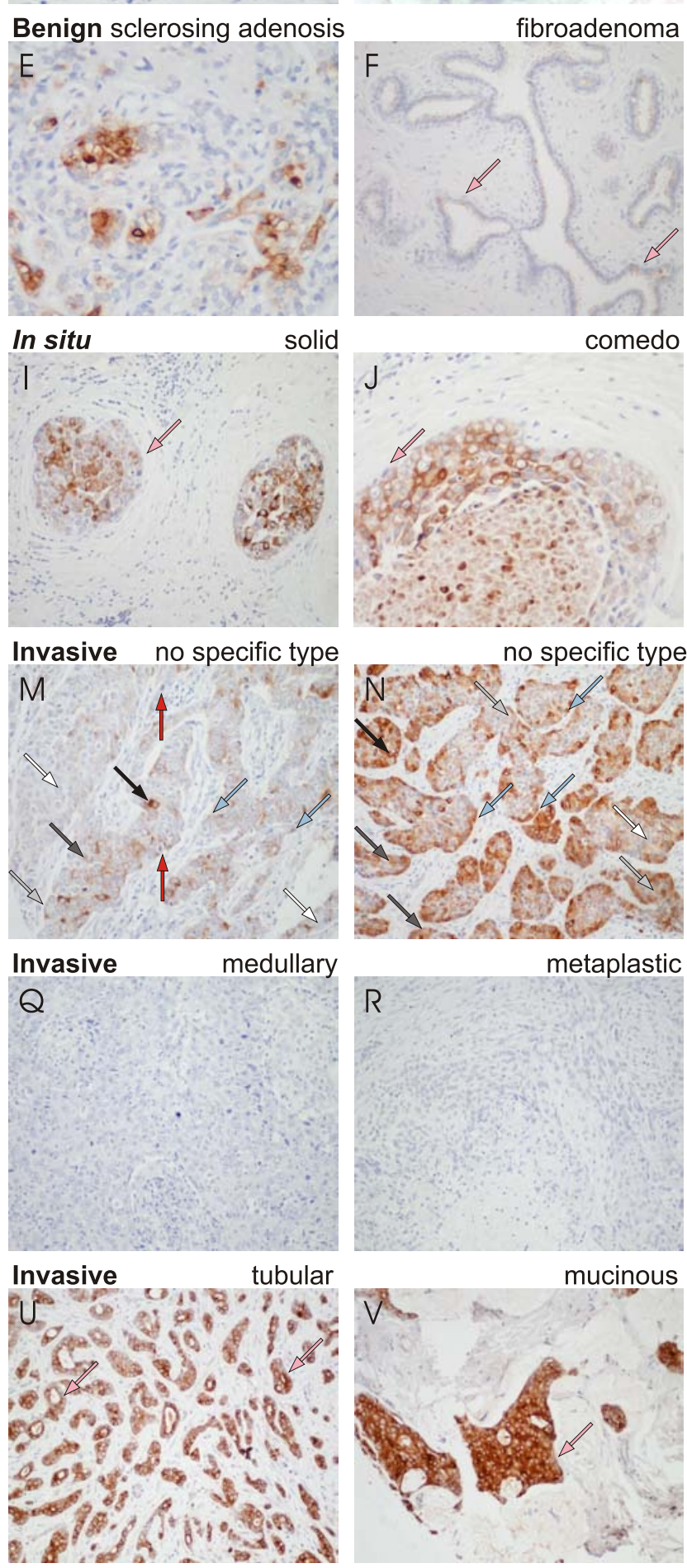
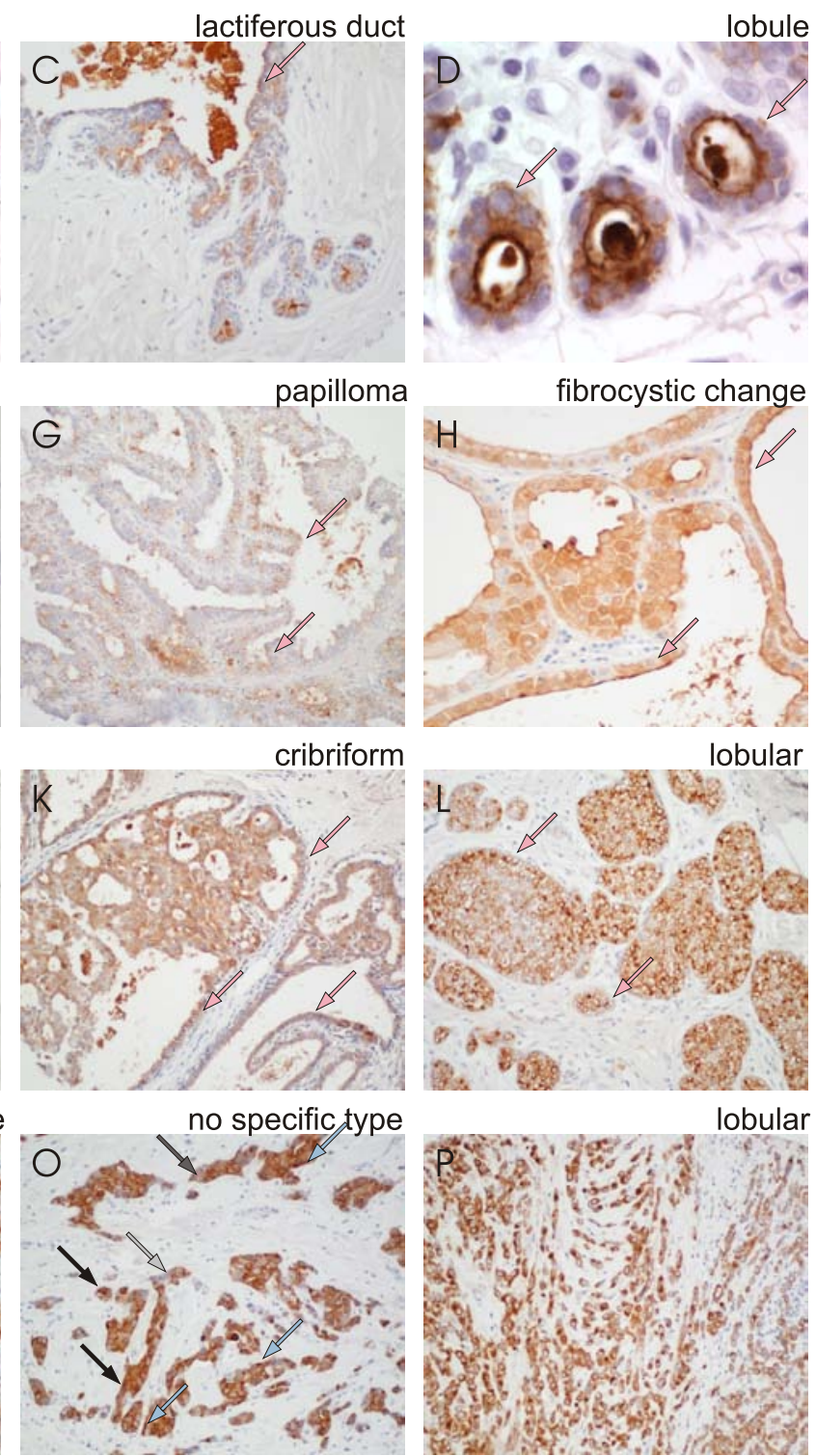
cribriform
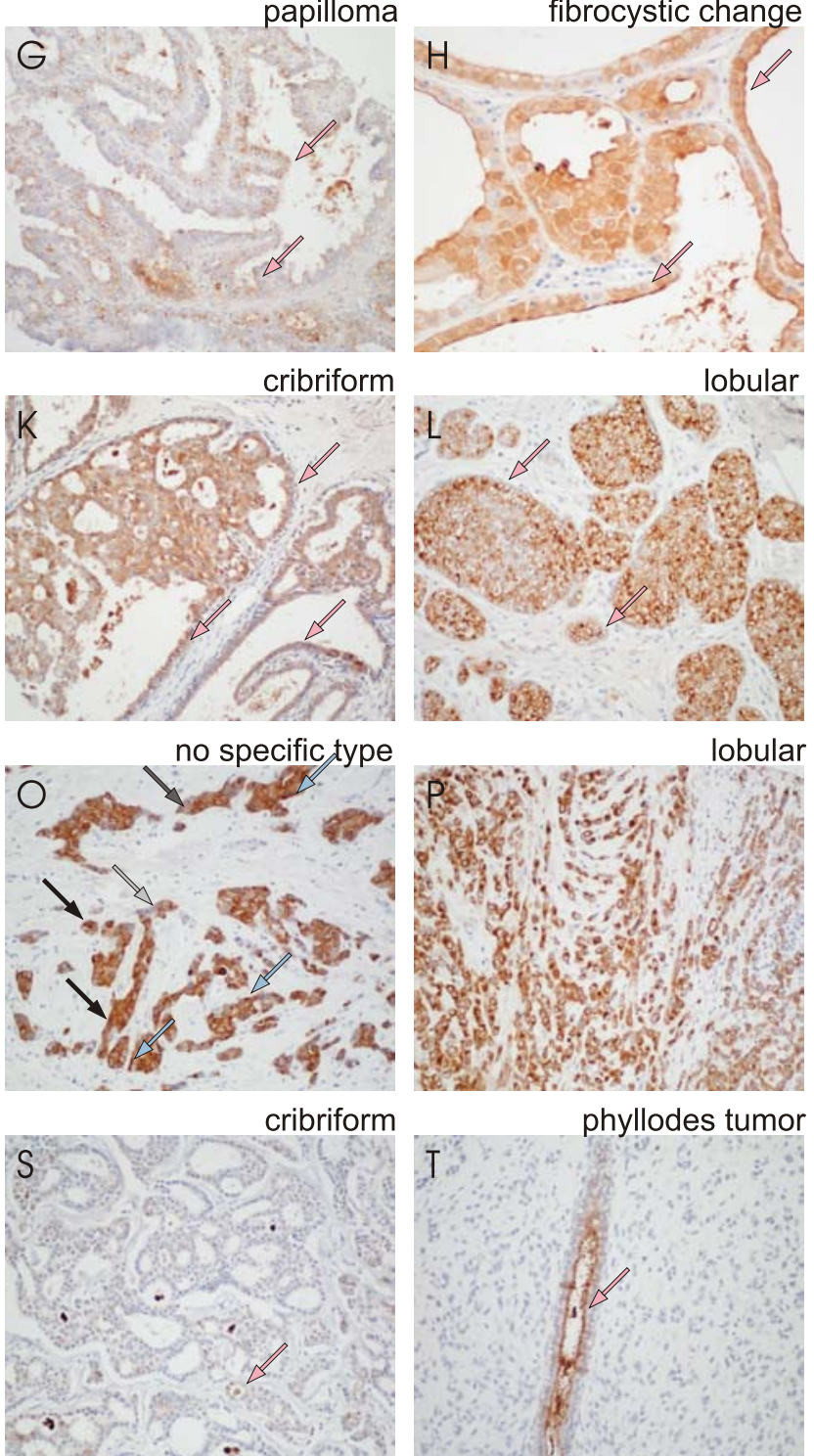

phyllodes tumor
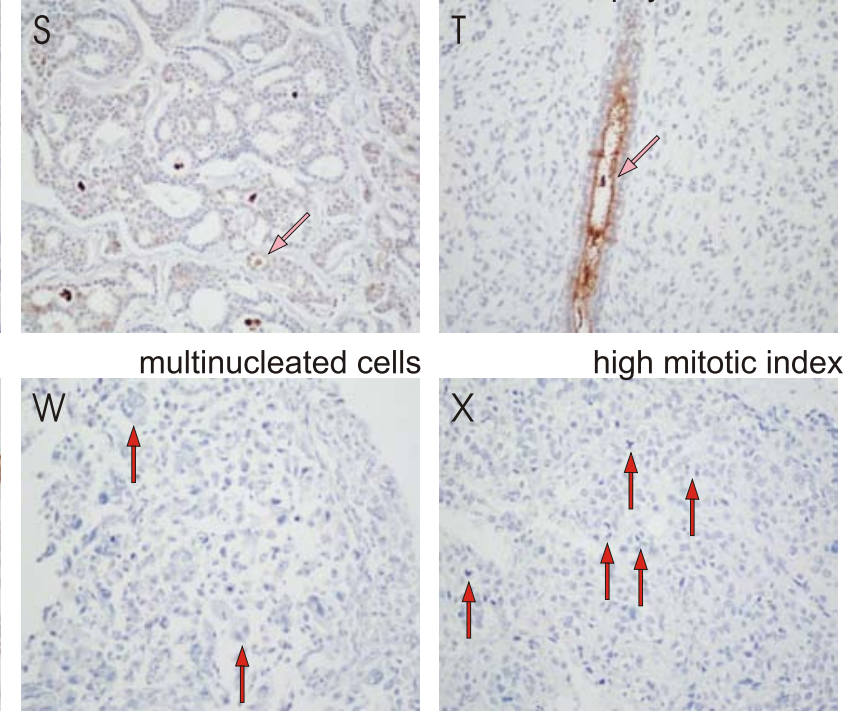
ables was evaluated by binary logistic regression and refined by stepwise selection.

\section{Results \\ Expression of TFF3 Protein in Normal Breast Epithelium}

The expression of TFF3 protein was characterized in normal breast tissue from 34 premenopausal and perimenopausal women. There was strong TFF3 immunoreaction in the cytoplasmic compartment of normal breast lobular epithelial cells which showed frequently clear cellular polarity (Figure 1, A and D). TFF3 protein was localized toward the luminal borders of the acini and was often absent at the basal membrane, which is consistent with TFF3 being secreted from the acini into the lumina of the lobules. No immunoreaction was observed in myoepithelial or stromal cells. TFF3 expression was detected in most of the normal breast lobules. TFF3 expression was detected also in epithelial cells of the intralobular and interlobular ducts (Figure 1B) and in the main lactiferous duct (Figure 1C). TFF3 was concentrated toward the lumina of the polarized epithelial cell monolayers. TFF3 has been measured in human milk, ${ }^{24}$ but the breast lobules in which TFF3 expression was detected in the present study were not from lactating women. These results show that TFF3 is synthesized in the normal nonlactating breast and are consistent with it being secreted from the breast epithelial cells and having a role in maintenance of the epithelial integrity of the lobular and ductal lumina.

We investigated the possibility that expression of TFF3 in the normal breast lobule might depend on cyclical changes that occur during the menstrual cycle. The morphological features of the breast which change during the menstrual cycle were assessed by histologic parameters. $^{25,26}$ The breast lobules in the two cases in which TFF3 expression was not detected were in the early follicular phase that is characterized by low circulating levels of estrogen and progesterone. Late follicular phase coincides with an increase in circulating estrogens and is characterized by clear acinar formation with distinct luminal cells. After ovulation and resultant increased circulating progesterone, the breast enters the early luteal phase, and lumina become enlarged compared with previous phases. The secretory phase is characterized by apocrine secretion from the luminal epithelial cells. The fifth, menstrual phase is associated with estrogen and progesterone withdrawal, and apocrine secretion abates. ${ }^{25} \mathrm{~A}$ trend toward elevated TFF3 ex- pression was observed in the late follicular phase compared with the early follicular phase, but the difference was not statistically significant.

The potential association between TFF3 expression and morphological changes that occur in breast lobular epithelial cells during the menstrual cycle was examined further in breast lobules of 40 younger women, aged between 18 and 39 years. Cytoplasmic expression of TFF3 in breast luminal epithelial cells was higher during late follicular and early luteal phases of the menstrual cycle (median, 560 and 430) when estrogen levels are higher than during the early follicular or late luteal phases (median, 25 and 110) when estrogen levels are low (Kruskal-Wallis II test, $P<0.01$ ). TFF3 immunoreaction was strong within the apocrine secretions of luminal cells in the secretory phase. During the menstrual phase, TFF3 immunoreaction was restricted mainly to the apical edge of the cells and residual secretory material within the lumina. The results indicate that there is an association between TFF3 expression in breast lobular epithelial cells and the stage of menstrual cycle.

\section{Alterations in TFF3 Expression in Benign Breast Lesions}

TFF3 was expressed in all 86 benign breast lesions examined (Figure 1, E-H). As with normal breast tissue, TFF3 protein was expressed only by the epithelial cells. Even in cases of sclerosing adenosis, in which the increased number of involved lobules are compressed and obliterated by stromal fibrosis (Figure 1E), the myoepithelial cells did not express TFF3. The intensity of the immunoreaction varied considerably among the 14 cases of sclerosing adenosis studied from almost undetectable to relatively strong. In the 10 fibroadenomas studied, TFF3 expression was detected always, but the intensity of the immunoreaction varied markedly between cells (Figure 1F). Fibroadenomas in which the glandular component was dominant tended to express more TFF3 than those in which the stromal component was dominant. In the six intraductal papillomas (Figure 1G), a higher proportion of cells contained detectable levels of TFF3 than in fibroadenomas or cases of sclerosing adenosis. In fibrocystic disease (Figure 1H), all epithelial cells present in areas of fibrocystic change expressed detectable TFF3, and the immunoreaction was strong in most. TFF3 was detected always in the cytoplasm, and the immunoreaction was sometimes granular in appearance. When acini were visible, TFF3 was concentrated toward the lumina, and the immunoreaction was strong in areas in

\footnotetext{
Figure 1. Expression of TFF3 in normal and diseased human breast. Sections from human breast tissue were analyzed by immunohistochemistry with a specific anti-human TFF3 mouse monoclonal IgG as described in Materials and Methods. Representative examples of the immunoreaction with normal human breast lobules (A and D); an interlobular duct (B); a lactiferous duct (C); sclerosing adenosis (E); a fibroadenoma (F); a ductal papilloma $(\mathbf{G})$; fibrocystic change (H); ductal carcinoma in situ of solid, comedo, and cribriform types (I, J, and $\mathbf{K})$; lobular carcinoma in situ (L); invasive ductal carcinoma of no specific type (M-O); invasive lobular carcinoma $(\mathbf{P})$; medullary $(\mathbf{Q})$, metaplastic $(\mathbf{R})$, and cribriform carcinomas $(\mathbf{S})$; a phyllodes tumor $(\mathbf{T})$; tubular $(\mathbf{U})$ and mucinous $(\mathbf{V})$ carcinomas; and invasive ductal carcinoma of no specific type with cytological atypia and multinucleated cells (W) and with cytological atypia and numerous mitoses (X) are shown. Arrows indicate malignant epithelial cells with TFF3 expression absent (M and $\mathbf{N}$; white), weakly positive (M-O; pale gray), moderately positive (M-O; intense gray), and strongly positive (M-O; black). Examples of cells in which TFF3 expression is polarized either away from the lumen (pink arrows) or away from the stroma (blue arrows) are indicated. Multinucleated cells in a carcinoma with prominent cytologic atypical (W), and mitotic cells in a carcinoma with mild cytologic atypical $(\mathbf{X})$ and in a ductal carcinoma of no specific type $(\mathbf{M})$ are indicated (red arrows). Original magnification: $\times 200(\mathbf{A}, \mathbf{C}, \mathbf{F}-\mathbf{X}) ; \times 400(\mathbf{E})$, and $\times 630(\mathbf{B}$ and $\mathbf{D})$.
} 
which cellular release of large secretory granules was apparent. The luminal release of TFF3 was particularly evident in apocrine metaplasia. TFF3 expression was especially high in cells that exhibit apocrine metaplasia within areas of fibrocystic change but was absent in squamous metaplasia.

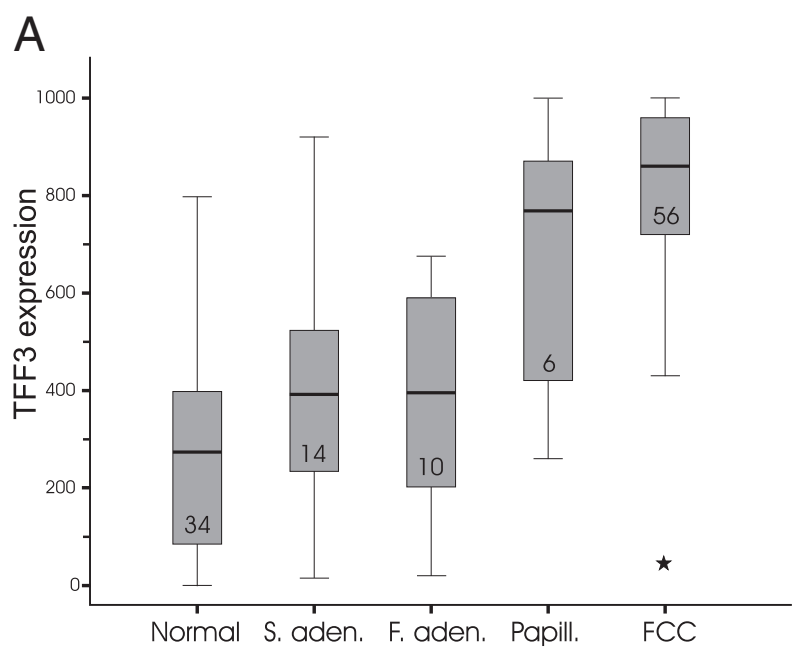

B
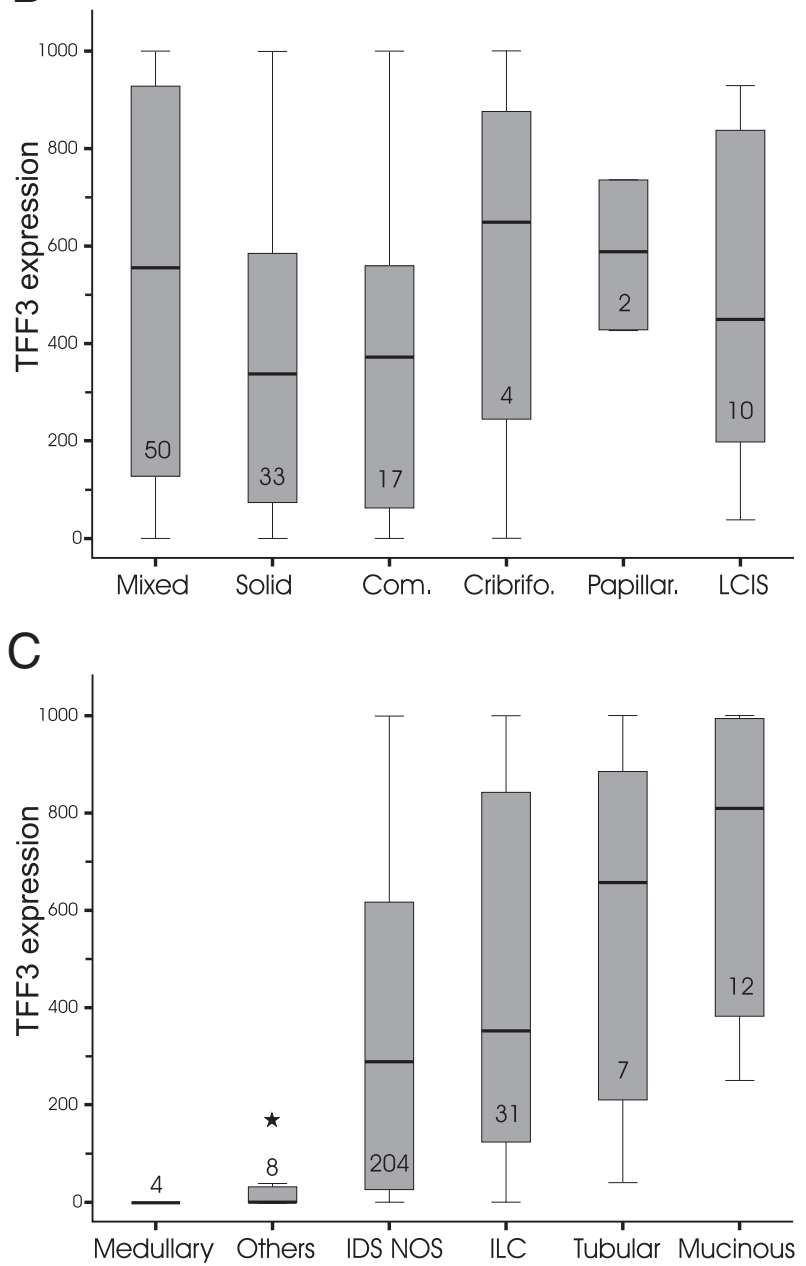

TFF3 expression in the normal breast tissues and in benign breast lesions was quantified to generate a histoscore (Figure 2A). Four cases each of sclerosing adenosis (29\%), fibroadenoma (40\%), and papilloma (67\%) and 53 cases of fibrocystic change (95\%) had high histoscores. TFF3 expression is significantly higher in papilloma of the breast and in areas of fibrocystic change than in normal breast tissue (Mann-Whitney $U$-test, $P<0.01$ and $P<0.001$, respectively). TFF3 expression in papilloma and in fibrocystic change is higher also than in sclerosing adenosis and fibroadenoma (Kruskal-Wallis II test, $P<0.001$ ).

\section{Expression of TFF3 in in Situ Malignant Breast Lesions}

TFF3 was detected in 93 of the 106 cases (88\%) of ductal carcinoma in situ studied, which comprised 50 of mixed histologic type, 33 solid, 17 comedo, 4 cribriform, and 2 papillary tumors (Figure 1, I-K). TFF3 immunoreaction was generally strong and, where cellular polarity was evident, was concentrated at the luminal edge but was distributed evenly throughout the cytoplasm in nonpolarized malignant epithelial cells. No statistically significant differences were observed in TFF3 expression between subtypes of carcinoma in situ (Figure 2B) or between different grades; 81 lesions were of high grade, 13 of intermediate grade, and 12 of low grade. TFF3 immunoreaction was detected in all cases of lobular carcinoma in situ (Figure 1L).

\section{Expression of TFF3 in Invasive Malignant Breast Lesions}

TFF3 was detected in 215 of 266 invasive tumors (Figure 1). The immunoreaction was cytoplasmic with a tendency toward perinuclear condensation in some cells, consistent with the concentration of the TFF3 protein in the Golgi apparatus before its secretion. Most of the immunoreactive tumor cells were moderately to strongly positive. TFF3 was not detected in the surrounding stromal, endothelial, inflammatory, or muscle cells. Malignant cells in tumors with strong TFF3 expression were scattered diffusely throughout the stroma (Figure 10), whereas those

\footnotetext{
Figure 2. Association of TFF3 expression with differentiation in diseased human breast. TFF3 expression was measured by immunohistochemistry in 34 samples of normal breast tissue, 14 lesions of sclerosing adenosis ( $\mathrm{S}$. aden.), 10 fibroadenomas (F. aden.), 6 ductal papillomas (Papill.), and 56 cases of fibrocystic change (FCC), and the relative levels of TFF3 expression were determined by derivation of a histoscore as detailed in Materials and Methods (A). Expression was measured also in 116 in situ malignant breast lesions (B) and in invasive breast tumors (C) from 266 patients. The horizontal bars represent the median values, the boxes indicate the range of the second and third quartiles of the data, and the whiskers represent the range of all of the data, that is, the sample minimum and the sample maximum. $\mathrm{x}$ The numbers refer to the number of cases analyzed for each type of lesion. The stars represent extreme values that are more than three times further from the second or third quartile than the difference between the second or third quartile and the median. Com., comedo; Cribrifo., cribriform; IDC NOS, invasive ductal carcinoma of no specific type; ILC, invasive lobular carcinoma; LCIS, lobular carcinoma in situ; Papillar., papillary.
} 
in tumors with weaker expression had a tendency to cluster or aggregate together within the stroma (Figure 1, $\mathrm{M}$ and $\mathrm{N}$ ). Within these clusters, malignant cells with higher TFF3 expression were concentrated on the edges of the cell aggregates and in some instances had polarized TFF3 immunoreaction that was more intense toward the stromal edge of the tumor cell.

TFF3 expression in the 204 invasive ductal carcinomas of no specific type (Figure 1, M-O) ranged from undetectable (not shown) to very high. In tumors with lower TFF3 expression (Figure 1, M and N), the intensity of the immunoreaction varied considerably between individual malignant cells. In tumors with very high TFF3 expression (Figure 10), most or all of the malignant cells were intensely immunoreactive. Variable levels of TFF3 expression were detected also in 31 cases of invasive lobular carcinoma (Figure 1P). Signet ring cells and those with a leading edge within lobular carcinomas had high TFF3 expression.

TFF3 was not detected in four cases of medullary carcinoma (Figure 1Q), and expression was generally low in the eight cases of the other special types of invasive ductal carcinoma. These comprised three micropapillary, two metaplastic (Figure 1R), one apocrine, and one cribriform (Figure 1S) carcinoma and one phyllodes tumor of the breast (Figure 1T). TFF3 expression is significantly higher in tubular (Figure 1U) and mucinous (Figure $1 \mathrm{~V}$ ) carcinomas, which are well differentiated and have a favorable prognosis, than in all other types of invasive breast carcinoma (Figure 2C, Kruskal-Wallis II test, $P<0.01)$. In mucinous carcinoma, the intracellular mucus and occasional extracellular mucus was immunoreactive for TFF3.

\section{Association of TFF3 Protein with Differentiation and Estrogen Response in Malignant Breast Epithelial Cells}

We investigated whether TFF3 expression is associated with histological grade of the tumors. All grade 1 tumors express detectable TFF3, but a third of grade 3 tumors contain no TFF3 protein (Figure 3A). The median values for TFF3 expression were 420 for grade 1 tumors, 340 for grade 2 tumors, and only 100 for grade 3 tumors; there is a significant negative association between tumor grade and TFF3 protein expression (Spearman's $\rho$ coefficient, $P<0.01)$. Thus, TFF3 expression is significantly lower in high-grade breast tumors than in low-grade tumors (Kruskal-Wallis II test, $P<0.001$ ).

Greater than $70 \%$ of the tumors in which TFF3 expression was not detected was grade 3 . To investigate if there was coordinate loss of expression of the paralagous trefoil protein, TFF1 in these tumors, TFF1 expression was analyzed by immunohistochemistry with an antibody raised against TFF1 protein. ${ }^{23}$ TFF1 immunoreaction was detected in only 2 of the 36 grade 3 tumors in which TFF3 expression was absent. These results indicate that there is coincident loss of TFF3 and TFF1 expression in breast cancer, which is consistent with interconnectivity of regulation of the TFF1 and TFF3 genes in breast cancer cells.
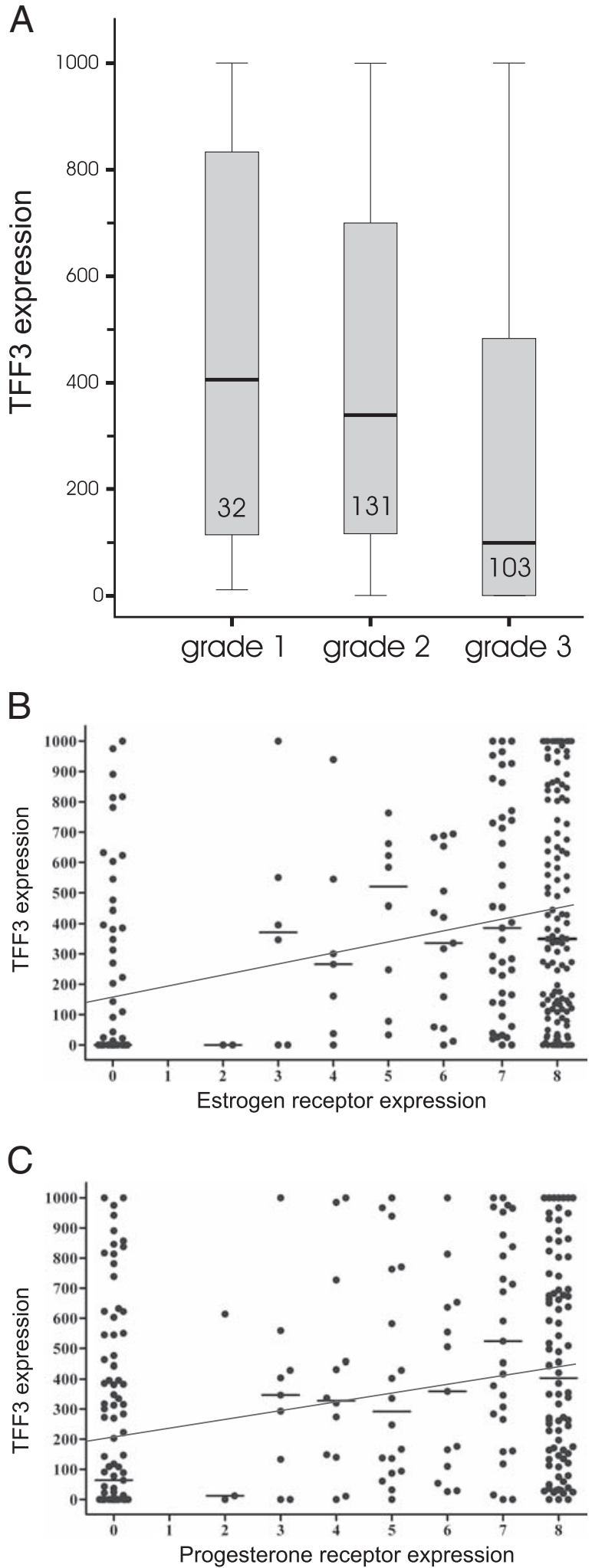

Figure 3. Association of TFF3 expression with pathological indicators of good prognosis. TFF3 expression determined by derivation of a histoscore was compared in tumors of different histologic grade (A) as described in the legend to Figure 2. The distribution of TFF3 expression in tumors with different Allred scores for estrogen receptor $(\mathbf{B})$ and progesterone receptor expression (C) are shown. Data points for samples with identical values are superimposed in the figure when there are more than three such data points. Horizontal bars indicate the median values of TFF3 expression. The sloping line is the regression line through the data. 
It is noteworthy that cases with a more anaplastic appearance, including a high nuclear-to-cytoplasmic ratio and absence of tubular differentiation, with cytologic atypia tended to express less TFF3 protein (Figure 1W), whereas TFF3 was expressed at markedly higher levels in invasive breast cancer types that are characterized by good differentiation: tubular and mucinous carcinomas. These observations coupled with the finding that TFF3 is secreted by normal breast acini suggest that the negative association of TFF3 expression with tumor grade is explained in part by its association with good differentiation.

Another possible reason for the strong negative association between TFF3 expression and tumor grade is that TFF3 expression is related to the proportion of mitotic cells. Cases with multinucleated cells or with a high mitotic incidence tended to express less TFF3 protein (Figure $1, W$ and $X)$. Cases with a low mitotic incidence tended to express more TFF3, and TFF3 was not detectable in dividing cells within tumors that do express TFF3 (Figure 1M). These observations of a negative association between TFF3 expression and mitotic incidence are exemplified by the absence of TFF3 protein in medullary carcinomas (Figure 1Q) that have a high mitotic index and by its higher level in tubular carcinomas (Figure $1 U$ ) that have a low mitotic index.

An association between expression of Ki-67, a nuclear nonhistone protein, and mitotic index has been reported by most researchers. ${ }^{27}$ To investigate further the potential relationship between TFF3 expression and proliferation of breast cancer cells, we measured expression of the proliferative biomarker Ki-67 by immunohistochemistry and investigated the association between TFF3 expression and Ki-67 expression. A strong negative correlation was observed between TFF3 protein and Ki-67 expression (Spearman's $\rho$ correlation, $P<0.001$ ), which agrees with the observations that tumors that express more TFF3 have low mitotic indices and is consistent with TFF3 expression militating against cell division in malignant breast epithelial cells.

TFF3 mRNA is induced by estrogen in estrogenresponsive breast cancer cells, ${ }^{20}$ and we investigated whether TFF3 protein expression is associated with estrogen receptor expression (Figure 3). Estrogen and progesterone receptors were measured during routine pathologic diagnosis and scored on the Allred scale from 0 to $8{ }^{28}$ The clinical decision to offer endocrine therapy is based on a cutoff value of 3.0. TFF3 expression was not detected in most of the tumors that do not express the estrogen receptor. A strong positive correlation was observed between estrogen receptor and TFF3 protein expression (Figure 3B, Pearson's correlation, $P<0.0001$ ) and between progesterone receptor and TFF3 protein expression (Figure 3C, Pearson's correlation, $P<$ $0.0001)$.

\section{Association between TFF3 Expression and Tumor Cell Infiltration}

The possible involvement of TFF3 in local invasion was considered first by evaluating TFF3 expression in meta- static cells. Tumor cells infiltrating underlying muscle expressed high levels of TFF3 (Figure 4, A and B). In cases with local epidermal invasion, the invading tumor cells often expressed high amounts of TFF3 (Figure 4C). TFF3 expression was detected in 9 of 10 cases of Paget disease of the nipple and was high in the majority (Figure 4D). Extremely high levels of expression of TFF3 were found in malignant epithelial cells involved in cancerization of the lobule (Figure 4, E and F), which is characterized by the migration of malignant cells from the original ductal carcinoma in situ lesion to the associated lobules without penetration of the myoepithelial cell layer. These observations led us to investigate whether tumors with overt locoregional invasion (Figure 5A) express higher levels of TFF3 than tumors without locoregional invasion. TFF3 expression was significantly higher in primary tumors associated with muscle invasion (Mann-Whitney $U$-test, $P<0.05$ ) and in tumor cells in Paget disease (Mann-Whitney U-test, $P<0.05)$ but not in tumors with skin invasion.

High TFF3 expression was observed in tumor cells invading the neural sheath (Figure 4, G and $\mathrm{H}$ ). We investigated whether high expression of TFF3 by tumor cells is associated with escape by the neural route. High TFF3 expression was observed in most cases in which neural invasion had been reported (Figure 5A) and was significantly higher than expression in invasive breast tumors without locoregional invasion (Mann-Whitney $U$-test, $P<0.05$ ).

Eighty-two of the 225 invasive tumors in which lymphovascular invasion was evaluated histologically by the presence of visible tumor cells within the tumor lymph or blood vessels showed evidence of lymphovascular invasion. High TFF3 expression was observed in tumor cells entering the lymphovascular system (Figure 4I). The expression of TFF3 in tumor cells of breast cancers in which intravasation of lymph and blood vessels was evident was higher than in tumor cells of cases in which intravasation was absent (Figure 5B, Mann-Whitney $U$-test, $P<$ 0.05). The ability of TFF3 expression in the primary tumor to predict any form of locoregional invasion, whether into muscle, skin, or via neurovascular bundles, was considered also. TFF3 expression was associated significantly with the presence of any type of invasion (Figure 5C, Mann-Whitney $U$-test, $P<0.05$ ).

The association of TFF3 protein expression with low histologic grade (Figure 3A) might obscure evaluation of its positive association with metastasis. We determined therefore the association between TFF3 expression and the presence of lymphovascular, or the presence of any form of invasion, within tumors of each histologic grade. The association between TFF3 expression and lymphovascular invasion in each tumor grade is shown in Figure 5D. Expression of TFF3 appears higher in tumors with associated lymphovascular invasion than in tumors without associated lymphovascular invasion in all tumor grades and reaches statistical significance in grade 3 tumors; high-grade tumors that express low levels of TFF3 are much less likely to invade the lymphovasculature (Mann-Whitney $U$-test, $P=0.013$ ). The association between higher TFF3 expression and any form of inva- 


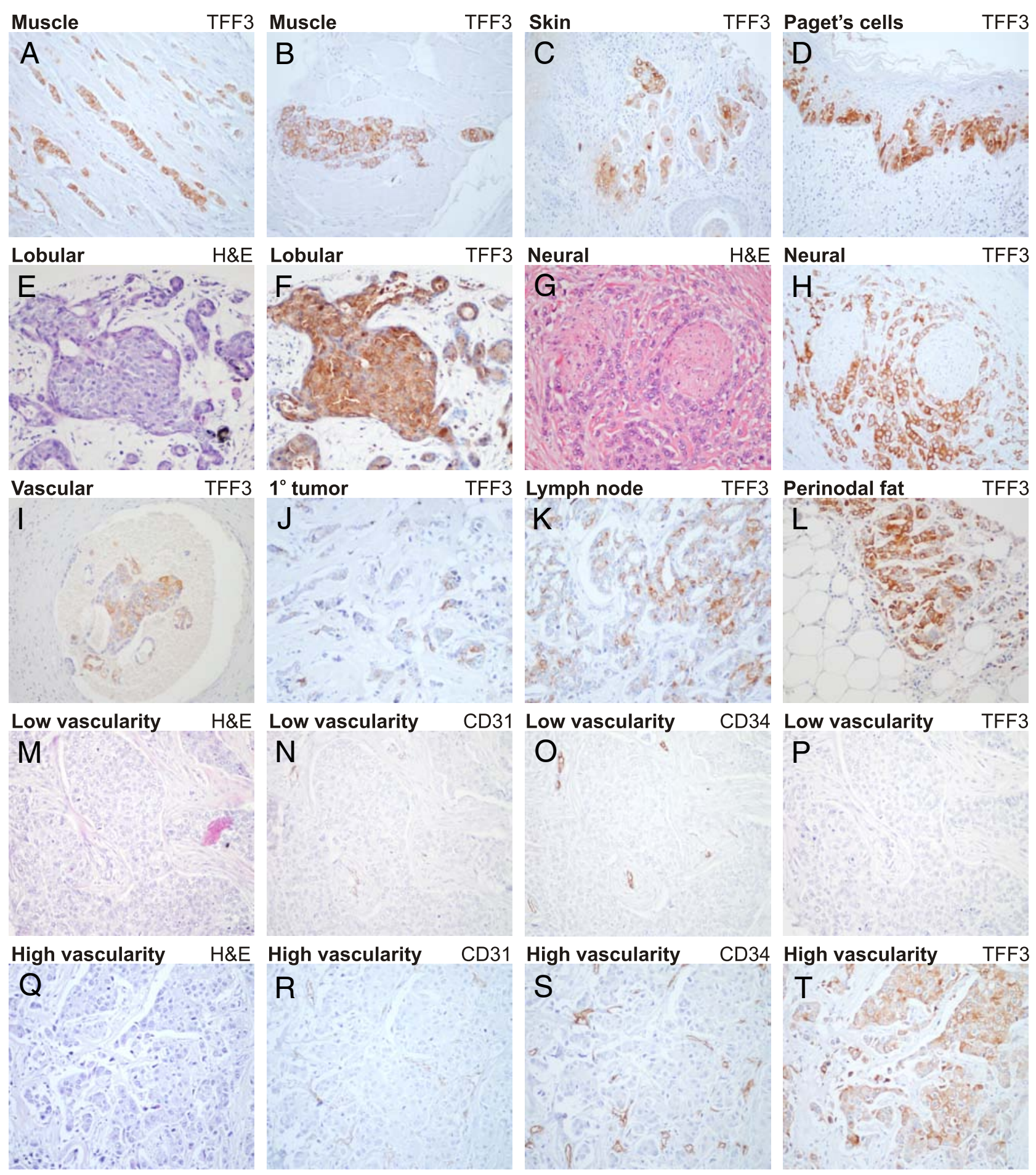

Figure 4. Expression of TFF3, CD31, and CD34 in invasive breast cancer. Sections of invasive breast cancer tissue were analyzed by immunohistochemistry with a specific anti-human TFF3 mouse monoclonal IgG as described in Materials and Methods. Representative examples of the immunoreaction with tumor cells invading muscle ( $\mathbf{A}$ and $\mathbf{B})$ and skin $(\mathbf{C})$, of Paget disease of the nipple $(\mathbf{D})$, of lobular cancerization by tumor cells $(\mathbf{E}$ and $\mathbf{F})$, of tumor cells invading neural bundles $(\mathbf{G}$ and $\mathbf{H}$ ), and the lymphovasculature (I) are shown. An example of TFF3 expression in a primary tumor, an associated lymph node, and the perinodal fat from the same case are shown (J-L). Examples of an invasive tumor with low vascularization $(\mathbf{M}-\mathbf{P})$ and high vascularization $(\mathbf{Q}-\mathbf{T})$ are shown. Sections were stained with H\&E (E, G, M, and $\mathbf{Q})$ or processed to detect expression of TFF3 ( $\mathbf{A}-\mathbf{D}, \mathbf{F}, \mathbf{H}-\mathbf{L}, \mathbf{P}$, and $\mathbf{T}), \mathrm{CD} 31(\mathbf{N}$ and $\mathbf{R})$, or CD34 $(\mathbf{O}$ and $\mathbf{S})$. Original magnification, $\times 200$.

sion in tumors of different histologic grade approached statistical significance in grade 1 and grade 2 tumors (Mann-Whitney $U$-test, $P=0.057$ and $P=0.091$, respectively) and was highly significant for grade 3 tumors $(P<0.01)$.

\section{TFF3 Expression in Lymph Node Metastasis}

We investigated whether expression of TFF3 in primary breast cancers is associated with lymph node metastasis. It has been reported previously that TFF1 expression 
does not or does predict axillary lymph node involvement. $^{9,29}$ Histologic assessment of the presence of breast cancer cells in the axillary lymph nodes was available for 201 cases of invasive breast cancer of which 84 had involved lymph nodes. TFF3 expression was higher
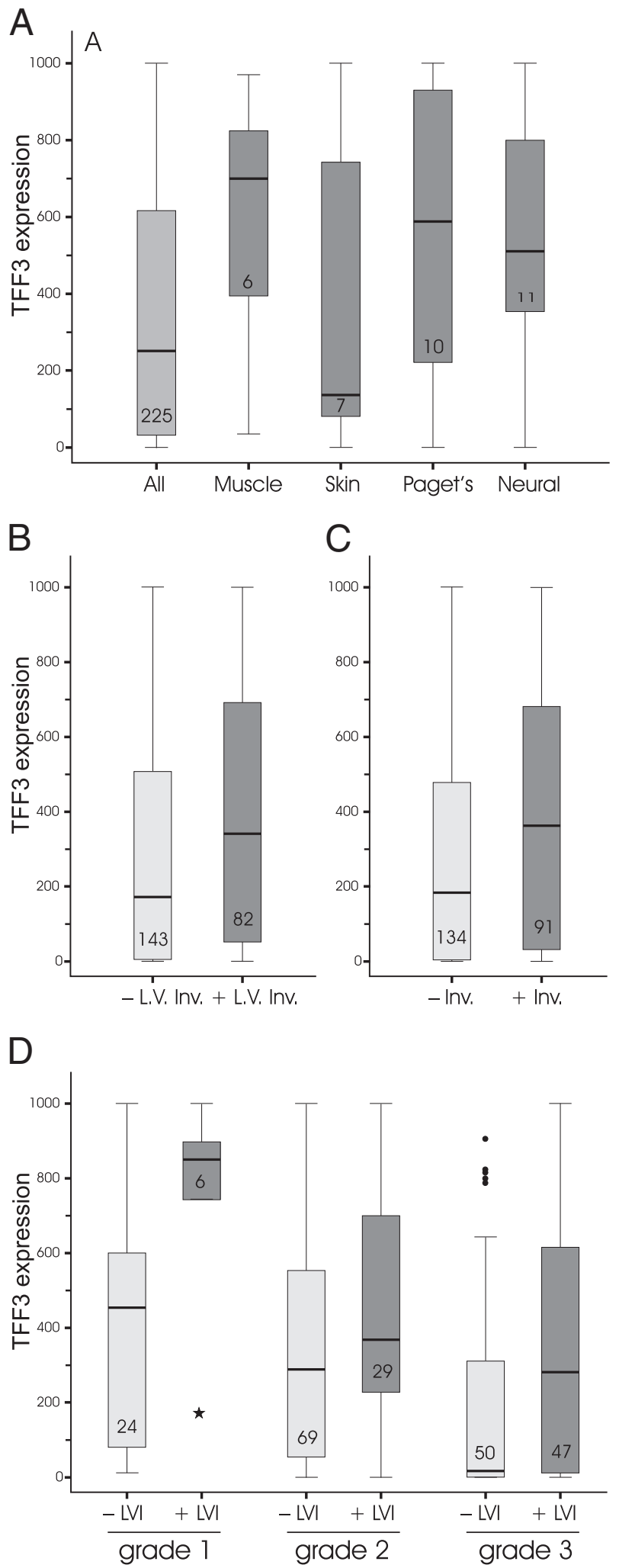

in primary tumor cells in tumors with associated involved lymph nodes than in tumors without lymph node metastases (Figure 6A, Mann-Whitney $U$-test, $P<0.05$ ), and higher TFF3 expression is associated with a larger number of involved lymph nodes (Pearson's correlation, $P<0.05$ ).

The levels of TFF3 expression in the primary tumor and in the corresponding metastatic cells in the lymph nodes are associated strongly (Figure 6C, Pearson's correlation, $P<0.0001$ ), and, in turn, expression in the metastatic tumor cells within the lymph nodes is maintained in malignant cells in the perinodal fat (Pearson's correlation, $P<0.001$ ). Although these correlations are highly significant, it appeared that there was an increase in TFF3 expression as the tumor cells moved along the metastatic cascade (Figure 6, B and C) from the primary tumor (Figure $4 \mathrm{~J}$ ) to the axillary lymph node (Figure 4K) to the perinodal fat (Figure 4L). TFF3 protein expression is associated positively with this metastatic progression (Spearman's $\rho$ correlation, $P<0.0001$ ) and is higher in tumor cells within the involved lymph nodes than in the primary tumor and is increased further in tumor cells in the perinodal fat that have evaded the lymph node capsule (Figure 6, B and C, Kruskal-Wallis II test, $P<$ $0.0001)$. These observations suggest that there is a selection for higher TFF3 expression as the tumor cells move to more distal metastatic sites and are consistent with TFF3 playing an active role in metastasis.

\section{Association of TFF3 Expression with Angiogenesis}

It has been reported that TFF3 stimulates angiogenesis. ${ }^{7}$ We considered the possibility that stimulation of a tumor vasculature may be one way in which TFF3 promotes lymphovascular invasion of malignant breast epithelial cells. Expression of the endothelial cell markers CD31 and CD34 was measured by immunohistochemistry and the density of immunoreactive blood vessels was calculated as described in Materials and Methods. Tumors with an inflammatory reaction, granulation tissue, tumor necrosis, tumor sclerosis, or large areas of mucin were excluded from this analysis. CD34 expression gave a higher estimate of the blood vessel density than CD31, but there was a strong positive correlation between the density of microvessels assessed with the two markers

\footnotetext{
Figure 5. Association of TFF3 expression with locoregional metastasis. TFF3 expression determined by derivation of a histoscore as detailed in Materials and Methods in invasive tumors with different types of locoregional infiltration or with neural invasion is compared with TFF3 expression in all evaluable invasive tumors (A). TFF3 expression is compared between tumors without (-L.V.Inv.) or with lymphovascular invasion (+L.V.Inv.) (B) and between tumors with no infiltration ( - Inv.) and with any form of infiltration (+Inv.) (C). TFF3 expression was compared between tumors of different histologic grade in which lymphovascular invasion was not detected $(-$ LVI $)$ and tumors in which it was (+LVI) (D). The horizontal bars represent the median values, the boxes indicate the range of the second and third quartiles of the data, and the whiskers represent the range of all of the data. The numbers refer to the number of cases analyzed for each type of lesion. The circles represent outliers of $>1.5$ times, and the stars represent extreme values of $>3.0$ times further from the second or third quartile than the difference between the second or third quartile and the median.
} 

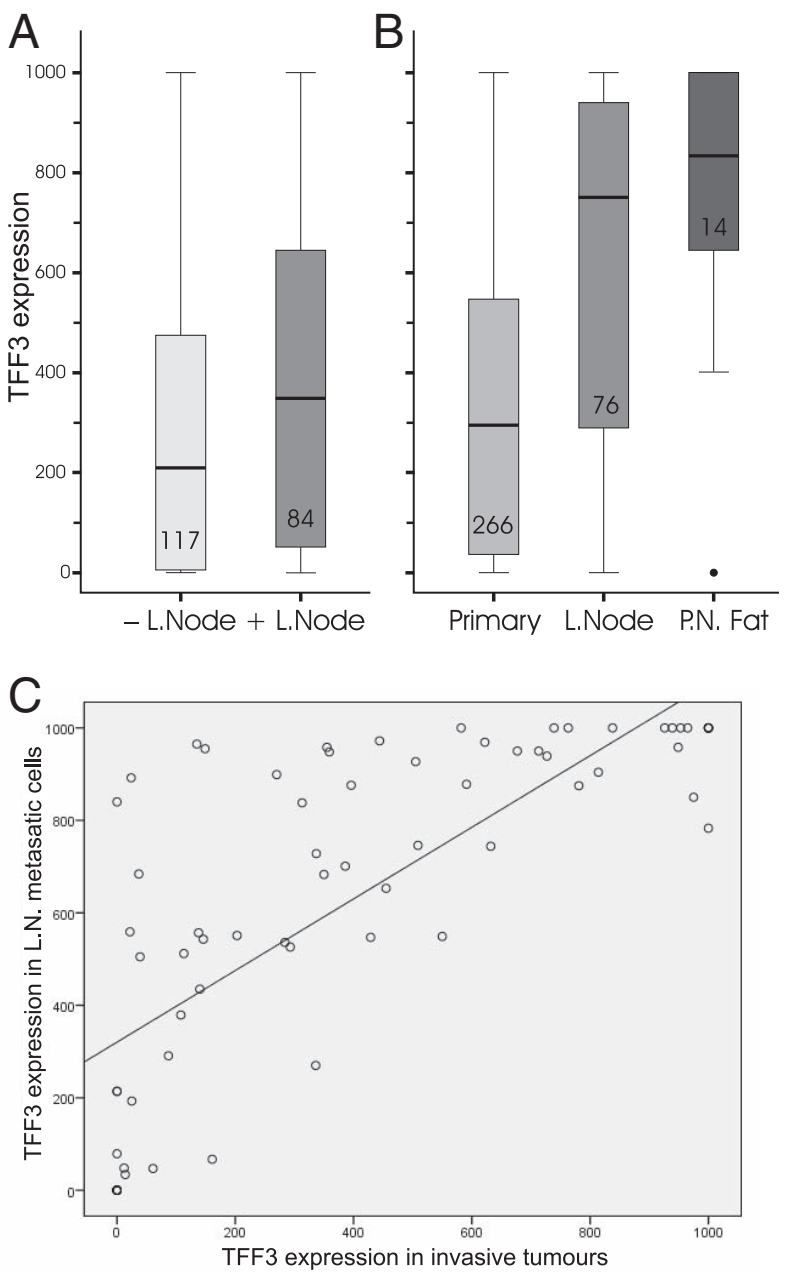

Figure 6. Association of TFF3 expression with lymph (L) node metastasis. TFF3 expression determined by derivation of a histoscore as detailed in Materials and Methods was compared in tumor cells from tumors without and with associated involved lymph nodes (A) and in tumor cells in invasive primary tumors, in involved lymph nodes, and in the perinodal (P.N.) fat (B). The horizontal bars represent the median values, the boxes indicate the range of the second and third quartiles of the data, and the whiskers represent the range of all of the data. The numbers refer to the number of cases analyzed for each type of lesion. The circle represents an outlier value that is $>1.5$ times further from the second quartile than the difference between the second quartile and the median. The association between TFF3 expression in invasive tumor cells in the primary tumors and in the associated involved lymph nodes is shown (C).

(Pearson's correlation, $P<0.01$ ). Tumors with low vascularity had relatively lower levels of TFF3 expression (Figure 4, M-P) than tumors with high vascularity (Figure 4, Q-T). High TFF3 expression was associated positively with blood vessel density as evaluated with both CD31 (Pearson's correlation, $P<0.05$ ) and CD34 (Pearson's correlation, $P<$ 0.01 ) endothelial markers. This finding is consistent with the hypothesis that TFF3 stimulates angiogenesis in vivo.

\section{Predictive Value of TFF3 as a Biomarker of Breast Cancer Metastasis}

At diagnosis, several pathologic and clinical factors, including lymphovascular invasion and lymph node status, contribute to clinical staging and inform clinical manage- ment and evaluation of prognosis. If TFF3 is important in breast cancer invasion and metastasis, it might be expected to be a predictor of lymphovascular invasion and lymph node metastasis. We evaluated the potential of TFF3 as an effective predictive biomarker of lymphovascular invasion and of lymph node metastasis by simple logistic regression analysis and its relative importance by multivariate binary logistic regression analysis. Binary logistic regression modeling is a multivariate technique that allows the probability of a defined outcome to be estimated by analysis of several parameters, including clinicopathologic features and molecular biomarkers.

In univariate analysis, TFF3 was a statistically significant predictor of lymphovascular invasion $(P<0.05)$. Multivariate binary logistic regression analysis found that tumor grade had the greatest ability to predict the presence of lymphovascular invasion amongst all of the factors considered ( $P=0.001$ to 0.004 ), followed closely by TFF3 ( $P=0.001)$. None of the other factors considered, age, tumor size, tumor type, estrogen receptor status, progesterone receptor status, CD31 expression, or CD34 expression, made a significant contribution to the prediction.

The regression equations were as follows. For Grade 1 tumors, $Z=-0.389+0.156$ (TFF3 expression) + $(-1.335)$. For Grade 2 tumors, $Z=-0.389+0.156$ (TFF3 expression $)+(-0.815)$. For Grade 3 tumors, $Z=-0.389+$ 0.156 (TFF3 expression) +0 .

TFF3 expression predicted lymph node metastasis as a univariate biomarker $(P<0.05)$. The ability of the nine variables, age, tumor type, tumor size, tumor grade, estrogen receptor status, progesterone receptor status, microvessel density evaluated by CD31 and CD34, and TFF3 expression, to predict lymph node metastasis was tested by multivariate binary logistic regression analysis. Tumor size $(P=0.001)$, tumor grade $(P=0.002-0.03)$, and TFF3 $(P=0.004)$ were retained in the model.

The regression equations were as follows. For Grade 1 tumors, $Z=-1.093+0.149$ (TFF3 expression) +0.022 (tumor size $)+(-1.231)$. For Grade 2 tumors, $Z=-1.093+$ 0.149 (TFF3 expression) + 0.022 (tumor size) $+(-1.092)$. For Grade 3 tumors, $Z=-1.093+0.149$ (TFF3 expression) +0.022 (tumor size) +0 .

These analyses show that TFF3 expression is an independent predictive biomarker of both lymphovascular invasion and lymph node metastasis and that it has greater predictive power than the other molecular markers analyzed.

\section{Discussion}

This is the first study of TFF3 protein expression in the human breast. Our results establish that TFF3 protein is present in normal breast epithelial cells; its cellular location indicates that it is secreted into the lumina of breast lobules and ducts. By analogy to the function of trefoil proteins in other tissues, the function of TFF3 in the normal human breast may be to protect and maintain the integrity of the epithelial surface. The other single domain trefoil protein TFF1 is expressed also by normal breast 
epithelial cells, ${ }^{30}$ which means that the breast epithelial cell is one of very few normal cell types in which TFF1 and TFF3 are co-expressed. ${ }^{19}$

TFF3 protein is expressed in most of the benign and malignant breast lesions studied. A strong association was observed between TFF3 expression and tumor differentiation; better differentiated tumor types express higher levels of TFF3. TFF3 protein expression was associated with expression of estrogen and progesterone receptors and with lower tumor grade, all of which are features of well-differentiated invasive breast cancer. These associations are consistent with our finding that TFF3 is a protein of normal breast epithelium and suggest that during the process of carcinogenesis and dedifferentiation TFF3 expression is lost from a subset of tumors.

Several functions have been ascribed to TFF3 on the basis of laboratory studies, including inhibition of gastric cancer cell growth, ${ }^{31}$ stimulation of angiogenesis in human umbilical vein endothelial cell and embryonic chicken cell models, ${ }^{7}$ and stimulation of invasion of different types of cells. ${ }^{6}$ Our study was designed to determine whether the profile of expression of TFF3 in vivo supports the hypothesis that TFF3 has these functions in malignant breast tumors. The negative association between TFF3 expression and tumor grade would be consistent with an inhibitory effect of TFF3 on breast cancer cell growth because the number of mitotic bodies is one of the three parameters that contribute to histologic tumor grade. Evidence for a negative effect of TFF3 on proliferation derives also from the observation that it is not expressed in tumors characterized by high mitotic indices and that it is absent from dividing cells within tumors that do express TFF3. Further investigation found a strong negative association between TFF3 protein expression and expression of the proliferation biomarker Ki-67.

Our data support the hypothesis that TFF3 stimulates angiogenesis in breast tumors. A positive association was observed between TFF3 expression and microvessel density, evaluated by the endothelial markers CD31 or CD34. An association between TFF3 expression and microvessel density, evaluated by CD34 expression, has been reported in gastric cancer..$^{12}$ In contrast to the present study, TFF3 expression was detected in endothelial cells and in the nuclei of epithelial cells in gastric tumor tissue. ${ }^{12}$ It is unclear if these anomalies are explained by tissue or technical differences.

A striking finding of our study is the strength and consistency of the association between TFF3 expression and a more metastatic phenotype in invasive breast cancer. TFF3 was expressed at higher levels in primary tumors with associated metastasis, and the expression of TFF3 was higher in malignant cells that have metastasized away from than in those within the primary tumor. Higher TFF3 expression was associated with invasion of muscle, the nipple, the neural sheath, and the lymphovasculature. It was associated also with lymph node positivity, the number of involved lymph nodes, and tumor cell escape from axillary lymph nodes by capsular invasion. Consistent with the hypothesis that tumor cells that express higher levels of TFF3 are more invasive, tumor cells that expressed high amounts of TFF3 were more likely to be dispersed through the stroma, whereas, in tumors with lower TFF3 expression, tumor cells were present as clusters within the stroma with the cells that expressed more TFF3 concentrated at the periphery of the tumor cell clusters.

Our data are consistent with a role of TFF3 in more than one of the different stages of metastasis reviewed by Nyuyen et al. ${ }^{32}$ During metastasis initiation, TFF3 enhances invasion by tumor cells of the stroma, in part by stimulating their motility, and attracts a more supportive stroma by increased angiogenesis. During metastasis progression, TFF3 increases penetration of the neural sheath and intravasation of the lymphovasculature. The association between TFF3 expression and the colonization of and subsequent escape from axillary lymph nodes indicates continued involvement of TFF3 early during metastasis virulence. The role of TFF3 protein expression in breast cancer metastasis has not been studied previously, but TFF3 was identified as the second highest ranking gene for the prediction of subsequent bony metastasis in an expression microarray study of early breast cancer, ${ }^{33}$ which is consistent with our finding that tumor cells that express higher levels of TFF3 are more likely to metastasize and suggests continued involvement of TFF3 later during metastasis virulence to facilitate bone colonization.

Interestingly, there appears to be a switch in the normal polarized secretion of TFF3 in invasive cancer. TFF3 is secreted toward the lumen away from the myoepithelial cell layer and stroma in normal breast epithelial cell monolayers (Figure 1, A-D). In well-differentiated breast tumors, polarized TFF3 expression and secretion away from the stroma into vestigial ductlike lumina is retained (Figure 1, I-L, S, U, and V). In poorly differentiated tumors, there appears to be a reversal in the epithelial cell polarization with TFF3 concentrated at the stromal face of the tumor cell clusters (Figure 1, M-O). This concentration of TFF3 toward the stroma suggests that TFF3 is secreted from the tumor cells into the stroma, which should allow it to exert any invasion-promoting effects in an autocrine fashion or by a paracrine mechanism on adjacent invasive malignant breast epithelial cells. Secretion of TFF3 into the stroma would facilitate also paracrine regulation of stromal cells, including potentially angiogenic effects on endothelial cells within the stroma.

Functional data from in vitro experiments that support our findings would be of value. It may be difficult to substantiate directly our in vivo findings because the TFF3 cellular polarity may depend on the existence of the structural architecture that is present within the breast tissue. Individual malignant breast epithelial cells that have potentially reversed polarity at the periphery of islands of invasive malignant cells would have to be isolated, and their polarity in vitro analyzed subsequently. There are, however, elegant three-dimensional models of breast cancer available in which cells become polarized. It would be of interest to study different malignant breast epithelial cells to investigate whether it is possible to detect alterations in polarized secretion in cells with different invasive potential. The complexities inherent in replication of our observations in vitro do not detract from the 
potential importance of our finding that the directional polarity of TFF3 secretion changes with loss of tissue architecture in some breast cancers.

TFF3 is expressed in normal breast epithelia, and we suggest that TFF3 is regulated by estrogen and has beneficial properties in normal breast epithelia. We propose that, early during breast tumorigenesis, TFF3 retains its association with normal functionality of breast epithelial cells and hence with differentiation. Subsequently, with loss of breast tumor cell differentiation, loss of normal intercellular contacts, and normal epithelial cell polarization, expression of TFF3 with its antimitotic, proangiogenic, and proinvasive properties is subverted to promote cessation of growth, development of tumor vasculature, intravasation and extravasation of tumor cells via the lymphovasculatur, and locoregional infiltration. An analogous change from beneficial to detrimental properties has been suggested for TFF1 in gastric cancer. ${ }^{34}$

The simple model for the effects of estrogens in breast cancer that accounts for the beneficial effects of estrogen antagonists and aromatase inhibitors is that estrogens control a set of genes that promote tumor progression through a variety of pathways. Although numerous studies have identified estrogen-responsive genes, the key genes that mediate the various effects of estrogens in breast cancer have yet to be defined. TFF3 may be one such mediator. Its expression is associated closely with that of the estrogen receptor in breast tumors and is induced by estrogens in breast cancer cells. ${ }^{20}$ The present study shows that the level and pattern of expression of TFF3 is consistent with a role in invasion and metastasis. The paradox remains, however, for both the estrogen receptor and TFF3 that they are expressed in normal breast epithelial cells and contribute, therefore, to the normal physiology of the breast epithelium but yet are involved in the progression of cancer. For the estrogen receptor that is a regulator of transcription, the repertoire of genes whose expression is controlled by estrogen may change during cellular transformation so that genes that are not regulated in the normal epithelium become regulated in cancer. ${ }^{35,36}$ In the case of TFF3, a different mechanism must be involved. It could be envisaged, for example, that the switch from a beneficial to a malign function may result from the breakdown of tissue architecture together with matrix remodeling which posits the epithelial cells adjacent to fibroblasts, muscle, nerve, and endothelial cells in an invasive tumor, whereas in the normal breast, epithelial cells are surrounded exclusively by myoepithelial cells. The importance of the compartmentalization provided by the myoepithelial cells is recognized widely and has led to them being referred to as the tumor suppressor cells of the mammary gland. The inversion of cell polarity observed in the present study will exacerbate the effects of the breakdown in tissue architecture because it will facilitate direct secretion of TFF3 into the ectopic location of the tumor stroma in which it will exert its biological effects in the stromal microenvironment.

The present study reinforces the view that TFF3 expression merits evaluation as a prognostic marker and as a predictive marker of response to therapy. Metastasis is largely responsible for mortality in breast cancer, and approved tests of prognosis include some genes thought to be involved in the metastatic process. TFF3 could not feature in currently approved gene expression profiles used to predict disease progression (eg, oncotype.dx), simply because it was not evaluated as a candidate gene. The possibility that TFF3 may be a useful marker of endocrine responsiveness in breast cancer was suggested initially by our report that estrogen induces expression of TFF3 mRNA in estrogen-responsive cells, ${ }^{20}$ has been supported by gene expression studies that found co-expression with estrogen receptor, ${ }^{21,22}$ and is reinforced by the significant association between TFF3 protein and estrogen receptor expression reported in the present study. In addition, TFF3 merits evaluation because it may be involved in mediating the adverse effects of estrogen on tumor progression. Tamoxifen inhibits the induction of TFF3 mRNA by estrogen, ${ }^{20}$ and it is probable that the malign effects of TFF3 will be mitigated by adjuvant endocrine therapy in women with hormone-responsive cancers. The usefulness of TFF3 as a marker of hormone responsiveness has not, however, been assessed and requires evaluation. It may also merit evaluation as a biomarker of response to other types of therapy such as adjuvant bisphosphonate therapy, ${ }^{37}$ given the predilection of tumors that express TFF3 to metastasize to bone. ${ }^{33}$

\section{Acknowledgments}

We thank members of the Pathology Department and Keiran O'Toole for advice and support for immunohistochemistry work and Katherine E. Mann and David V. McGeeney (School of Mathematics and Statistics, Newcastle University, Newcastle upon Tyne, United Kingdom) for their advice and guidance during the statistical analyses of our data.

\section{References}

1. May FEB, Westley BR: Trefoil proteins: their role in normal and malignant cells. J Pathol 1997, 183:4-7

2. Thim L, May FEB: Structure of mammalian trefoil factors and functional insights. Cell Mol Life Sci 2005, 62:2956-2973

3. May FEB, Church ST, Major S, Westley BR: The closely related estrogen-regulated trefoil proteins TFF1 and TFF3 have markedly different hydrodynamic properties, overall charge, and distribution of surface charge. Biochemistry 2003, 42:8250-8259

4. Muskett FW, May FEB, Westley BR, Feeney J: Solution structure of the disulfide-linked dimer of human intestinal trefoil factor (TFF3): the intermolecular orientation and interactions are markedly different from those of other dimeric trefoil proteins. Biochemistry 2003, 42:1513915147

5. Williams MA, Westley BR, May FEB, Feeney J: The solution structure of the disulphide-linked homodimer of the human trefoil protein TFF1. FEBS Lett 2001, 493:70-74

6. Emami S, Le Floch N, Bruyneel E, Thim L, May F, Westley B, Rio M, Mareel M, Gespach C: Induction of scattering and cellular invasion by trefoil peptides in src- and RhoA-transformed kidney and colonic epithelial cells. FASEB J 2001, 15:351-361

7. Rodrigues S, Van Aken E, Van Bocxlaer S, Attoub S, Nguyen QD, Bruyneel E, Westley BR, May FEB, Thim L, Mareel M, Gespach C, Emami S: Trefoil peptides as proangiogenic factors in vivo and in 
vitro: implication of cyclooxygenase-2 and EGF receptor signalling FASEB J 2003, 17:7-16

8. Lefebvre O, Chenard MP, Masson R, Linares J, Dierich A, LeMeur M, Wendling C, Tomasetto C, Chambon P, Rio MC: Gastric mucosa abnormalities and tumorigenesis in mice lacking the pS2 trefoil protein. Science 1996, 274:259-262

9. Foekens JA, Rio MC, Seguin P, van Putten WL, Fauque J, Nap M, Klijn JGM, Chambon P: Prediction of relapse and survival in breast cancer patients by pS2 protein status. Cancer Res 1990, 50:3832-3837

10. Thompson AM, Elton RA, Hawkins RA, Chetty U, Steel CM: PS2 mRNA expression adds prognostic information to node status for 6-year survival in breast cancer. Br J Cancer 1998, 77:492-496

11. Yamachika T, Werther JL, Bodian C, Babyatsky M, Tatematsu M, Yamamura Y, Chen A, Itzkowitz S: Intestinal trefoil factor: a marker of poor prognosis in gastric carcinoma. Clin Cancer Res 2002, 8:10921099

12. Dhar DK, Wang TC, Tabara H, Tonomoto Y, Maruyama R, Tachibana $\mathrm{M}$, Kubota $\mathrm{H}$, Nagasue $\mathrm{N}$ : Expression of trefoil factor family members correlates with patient prognosis and neoangiogenesis. Clin Cancer Res 2005, 11:6472-6478

13. Yio X, Zhang JY, Babyatsky M, Chen A, Lin J, Fan QX, Werther JL, Itzkowitz S: Trefoil factor family-3 is associated with aggressive behavior of colon cancer cells. Clin Exp Metastasis 2005, 22:157-165

14. Babyatsky M, Lin J, Yio X, Chen A, Zhang JY, Zheng Y, Twyman C, Bao X, Schwartz M, Thung S, Lawrence Werther J, Itzkowitz S: Trefoil factor-3 expression in human colon cancer liver metastasis. Clin Exp Metastasis 2009, 26:143-151

15. Masiakowski P, Breathnach R, Bloch J, Gannon F, Krust A, Chambon $P$ : Cloning of cDNA sequences of hormone-regulated genes from the MCF-7 human breast cancer cell line. Nucleic Acids Res 1982, 10: 7895-7903

16. May FEB, Westley BR: Cloning of estrogen-regulated messenger RNA sequences from human breast cancer cells. Cancer Res 1986, 46(12 Pt 1):6034-6040

17. May FEB, Westley BR: Identification and characterization of estrogenregulated RNAs in human breast cancer cells. J Biol Chem 1988, 263:12901-12908

18. May FEB, Semple JI, Prest SJ, Westley BR: Expression and motogenic activity of TFF2 in human breast cancer cells. Peptides 2004, 25:865-872

19. Poulsom R, Hanby AM, Lalani EN, Hauser F, Hoffmann W, Stamp GWH: Intestinal trefoil factor (TFF 3) and pS2 (TFF 1), but not spasmolytic polypeptide (TFF 2) mRNAs are co-expressed in normal, hyperplastic, and neoplastic human breast epithelium. J Pathol 1997, 183:30-38

20. May FEB, Westley BR: Expression of human intestinal trefoil factor in malignant cells and its regulation by oestrogen in breast cancer cells. J Pathol 1997, 182:404-413

21. Gruvberger $S$, Ringnér M, Chen $Y$, Panavally S, Saal LH, Borg A, Fernö M, Peterson C, Meltzer PS: Estrogen receptor status in breast cancer is associated with remarkably distinct gene expression patterns. Cancer Res 2001, 61:5979-5984
22. West M, Blanchette $\mathrm{C}$, Dressman $\mathrm{H}$, Huang E, Ishida S, Spang R, Zuzan H, Olson JA JR, Marks JR, Nevins JR: Predicting the clinical status of human breast cancer by using gene expression profiles. Proc Natl Acad Sci U S A 2001, 98:11462-11467

23. Westley BR, Griffin SM, May FEB: Interaction between TFF1, a gastric tumor suppressor trefoil protein, and TFIZ1, a Brichos domain-containing protein with homology to SP-C. Biochemistry 2005, 44:79677975

24. Vestergaard EM, Nexo E, Wendt A, Guthmann F: Trefoil factors in human milk. Early Hum Dev 2008, 84:631-635

25. Vogel PM, Georgiade NG, Fetter BF, Vogel FS, McCarty KS Jr: The correlation of histologic changes in the human breast with the menstrual cycle. Am J Pathol 1981, 104:23-34

26. Ramakrishnan R, Khan SA, Badve S: Morphological changes in breast tissue with menstrual cycle. Mod Pathol 2002, 15:1348-1356

27. Yerushalmi R, Woods R, Ravdin PM, Hayes MM, Gelmon KA: Ki67 in breast cancer: prognostic and predictive potential. Lancet Oncol 2010, 11:174-183

28. Allred DC: Issues and updates: evaluating estrogen receptor-alpha, progesterone receptor, and HER2 in breast cancer. Mod Pathol 2010, 23(Suppl 2):S52-S59

29. Henry JA, Piggott NH, Mallick UK, Nicholson S, Farndon JR, Westley BR, May FEB: Prediction of relapse and survival in breast cancer patients by pS2 protein status. Br J Cancer 1991, 63:615-622

30. Piggott NH, Henry JA, May FEB, Westley BR: Antipeptide antibodies against the pNR-2 oestrogen-regulated protein of human breast cancer cells and detection of pNR-2 expression in normal tissues by immunohistochemistry. J Pathol 1991, 163:95-104

31. Uchino $\mathrm{H}$, Kataoka $\mathrm{H}$, Itoh $\mathrm{H}$, Hamasuna R, Koono M: Overexpression of intestinal trefoil factor in human colon carcinoma cells reduces cellular growth in vitro and in vivo. Gastroenterology 2000, 118:60-69

32. Nguyen DX, Bos PD, Massagué J: Metastasis: from dissemination to organ-specific colonization. Nat Rev Cancer 2009, 9:274-284

33. Smid M, Wang Y, Klijn JGM, Sieuwerts AM, Zhang Y, Atkins D, Martens JWM, Foekens JA: Genes associated with breast cancer metastatic to bone. J Clin Oncol 2006, 24:2261-2267

34. May FEB, Griffin SM, Westley BR: The trefoil factor interacting protein TFIZ1 binds the trefoil protein TFF1 preferentially in normal gastric mucosal cells but the co-expression of these proteins is deregulated in gastric cancer. Int J Biochem Cell Biol 2009, 41:632-640

35. Wilson CL, Sims AH, Howell A, Miller CJ, Clarke RB: Effects of oestrogen on gene expression in epithelium and stroma of normal human breast tissue. Endocr Relat Cancer 2006, 13:617-628

36. Wright PK, May FEB, Darby S, Saif R, Lennard TWJ, Westley BR: Estrogen regulates vesicle trafficking gene expression in EFF-3. EFM-19 and MCF-7 breast cancer cells Int J Clin Exp Pathol 2009, 2:463-475

37. Diel IJ, Solomayer EF, Costa SD, Gollan C, Goerner R, Wallwiener D, Kaufmann M, Bastert G: Reduction in new metastases in breast cancer with adjuvant clodronate treatment. N Engl J Med 1998, 339:357-363 